\title{
Displacement Based Unsupervised Metric for Evaluating Rank Aggregation
}

\author{
Maunendra Sankar Desarkar, Rahul Joshi, and Sudeshna Sarkar \\ Department of Computer Science and Engineering, \\ Indian Institute of Technology Kharagpur, \\ Kharagpur, India - 721302 \\ \{maunendra, rahulrj, sudeshna\}@cse.iitkgp.ernet.in
}

\begin{abstract}
Rank Aggregation is the problem of aggregating ranks given by various experts to a set of entities. In context of web, it has applications like building metasearch engines, combining user preferences etc. For many of these applications, it is difficult to get labeled data and the aggregation algorithms need to be evaluated against unsupervised evaluation metrics. We consider the Kendall-Tau unsupervised metric which is widely used for evaluating rank aggregation task. Kendall Tau distance between two permutations is defined as the number of pairwise inversions among the permutations. The original Kendall Tau distance treats each inversion equally, irrespective of the differences in rank positions of the inverted items. In this work, we propose a variant of Kendall-Tau distance that takes into consideration this difference in rank positions. We study, examine and compare various available supervised as well as unsupervised metrics with the proposed metric. We experimentally demonstrate that our modification in Kendall Tau Distance makes it potentially better than other available unsupervised metrics for evaluating aggregated ranking.
\end{abstract}

Keywords: Information Retrieval, Rank Aggregation, Distance Metrics, Kendall Tau Distance.

\section{Introduction}

Rank aggregation problem combines the ranking of entities obtained from multiple ranking functions in order to produce an aggregate ranked list. The input rankings are referred to as base rankers, or experts. There are various scenarios where it is difficult to obtain labeled data and the rankings from different sources need to be aggregated. In such cases, aggregate ranking needs to be evaluated with the help of unsupervised metrics. Generally, in such cases, the resultant aggregate ranking is evaluated by average distance with all available rankings given by different experts. Probably the most widely used distance metric used for this purpose is Kendall Tau Distance. The Kendall Tau distance is a metric that counts the number of pairwise disagreements between two ranked lists. 
However, inversion of a document pair having higher rank distance should be relatively more severe than that of a document pair having lower rank distance. Kendall Tau distance doesn't treat inversions based on their displacement distance. To get better distance between two ranked lists, we can consider displacement distance, displacement position and position weights while computing distance. In this paper, we propose a variant of Kendall Tau distance which treat document pair inversions based on their displacement distance.

There are several supervised and unsupervised metrics for judging the performance of ranking and rank aggregation algorithms. Supervised metrics such as Precision, Recall, NDCG use ground truth information about document relevance for determining the quality of the algorithm. Most of the unsupervised metrics for rank aggregation are based on distances between two ranked lists. Kendall Tau Distance, Spearman Footrule are two such distance measures widely discussed in literature.

Several researchers 10218 have pointed out that it may not be suitable for evaluation of rank aggregation as it gives equal importance to all the pairs. [4] discusses of a modification to Spearman FootRule distance, called Canberra distance for ranked lists. Canberra distance considers element positions for computing the distance. Cost of inversions are high when the items are at the top in either of the lists. [5] extended both Spearman Footrule distance and Kendall Tau Distance and proposed new formulations of Kendall Tau distance and Spearman Footrule distance by taking into consideration element weights, position weights and pairwise distances between permutations. Each inverted pair $(i, j)$, bears a penalty of $w_{i} w_{j}$ where $w_{i}$ and $w_{j}$ are determined by the positions of the items $i$ and $j$ in the two lists. Getting element weight and similarities between items are difficult. So we concentrate on the version where $w_{i}$ s are set according to position weights.

We propose a variant of Kendall Tau Distance where in we penalize a document pair inversion if it has higher rank distance. This way, the overall Kendall Tau distance will be more if the list has document inversions with higher rank distance and less with lower rank distance. We propose such a metric and name it KTDispSq. We compare this metric with existing unsupervised metrics, and experimentally demonstrate KTDispSq is potentially better than other metrics.

\section{Some Existing Distance Measures}

Let $\tau_{1}, \tau_{2}$ be two lists. Also assume that $\tau_{1}(i)$ denotes the rank of item $i$ in $\tau_{1}$. We now give the definitions of few distance measures that are used for evaluating rank aggregation outputs.

- Kendall Tau Distance (KT): KT distance between two ranked lists is defined as the number of inversions, i.e. item pairs $(i, j)$ such that in one list $i$ appears before $j$, and in the other list $j$ appears before $i$. Formally, $K T\left(\tau_{1}, \tau_{2}\right)=\sum_{i<j} I_{i j}$. Value of $I_{i j}$ is 1 if $(i, j)$ is inverted and 0 otherwise. 
- Spearman Footrule (SF): This metric can be expressed as: $\sum_{i} \mid \tau_{1}(i)-$ $\tau_{2}(i) \mid$. SF is known to be a 2 -approximation of $\mathrm{KT}$.

- Canberra: Canberra distance 4 between two ranked lists $\tau_{1}$ and $\tau_{2}$ is defined as: $\sum_{i} \frac{\left|\tau_{1}(i)-\tau_{2}(i)\right|}{\left|\tau_{1}(i)\right|+\left|\tau_{2}(i)\right|}$. The denominator ensures that cost of inversions are high when the items are at the top in either of the lists.

- Position weighted Kendall Tau: We call this measure as PosWtD. It penalizes each inversion $(i, j)$ by $w_{i} w_{j}$ where $w_{i}$ and $w_{j}$ are determined by the positions of the items $i$ and $j$ in the two lists. Mathematically, $\operatorname{Pos} W t D\left(\tau_{1}, \tau_{2}\right)=\sum_{i<j} w_{i} w_{j} I_{i j}$. Details on how the weights are set can be found in [5].

\section{The Proposed Metric (KTDispSq)}

The proposed metric KTDispSq penalizes an inverted pair by an increasing function of the rank distances d1 and d 2 for the two lists. More formally, the cost of each inversion is defined as:

$$
\bar{K}_{i, j}\left(\tau_{1}, \tau_{2}\right)=\frac{d_{1}^{2}+d_{2}^{2}}{4 n^{2}}
$$

Here,

$-d_{1}=$ absolute rank distance between documents $i$ and $j$ in ranked list $\tau_{1}$

$-d_{2}=$ absolute rank distance between documents $i$ and $j$ in ranked list $\tau_{2}$

$-n=$ total number of documents in ranked lists $\tau_{1}, \tau_{2}$

This way, an inverted docuemnt pair $(i, j)$ with more rank distance in both lists gets penalized more and contributes more in computing distance. The distance between two ranked lists is computed as the sum of these penalties over all the inverted pairs.

Example: Consider two ranked lists as

$$
\tau_{1}=\left[\begin{array}{lllll}
1 & 4 & 3 & 2 & 5
\end{array}\right] \text { and } \tau_{2}=\left[\begin{array}{lllll}
1 & 5 & 3 & 4 & 2
\end{array}\right] .
$$

Here we have four inverted pairs as $(2,5)(3,4)(3,5)$ and $(4,5)$. Inverted pair weights for KTDispSq can be computed as below:

$$
\begin{aligned}
& -(2,5)=\frac{\left(1^{2}+3^{2}\right)}{4 * 5^{2}}=0.1 \\
& -(3,4)=\frac{\left(1^{2}+1^{2}\right)}{4 * 5^{2}}=0.02 \\
& -(3,5)=\frac{\left(2^{2}+2^{2}\right)}{4 * 5^{2}}=0.08 \\
& -(4,5)=\frac{\left(3^{2}+2^{2}\right)}{4 * 5^{2}}=0.13
\end{aligned}
$$

Hence, the total distance KTDispSq between the two lists is: $(0.1+0.02+$ $0.08+0.13)=.33$.

We later experimentally compare the performance of KTDispSq with that of some existing unsupervised measures such as KT, Canberra Distance [4, SF, and PosWtD [5]. 


\section{Experiments and Results}

In order to judge how good the proposed measure is, we propose two sets of experiments. Details of the experimental mechanisms, and the results are given in the following subsections.

We consider Borda 9, QuickSort [9, MC4 [3], CombScore 1] as rank aggregation methods along with Precision, MAP, Kendall Tau (KT), KTDisp, KTDispSq, Canberra 4, Spearman Footrule (SF) and PosWtD [5] as metrics. We worked on LETOR 4.0 MQ2007, MQ2008 and Yahoo LTRC datasets for all experiments. These datasets have querywise document scores for various features. We consider each feature as a ranker or an expert. Due to space constraints, we report results on MQ2007 dataset only.

\subsection{Correlation with Other Metrics}

In this experiment, we first try several unsupervised rank aggregation algorithms and evaluate them using the supervised evaluation metrics mentioned above. The unsupervised metrics compared in this paper are all distance metrics, and lower score indicates better quality of the aggregation result. On the other hand, for the supervised metric MAP, higher score indicates better performance. Hence, a good algorithm should get low score for the unsupervised metrics used in this paper, but high score for MAP. In other words, if we look at the scores given to the rank aggregation algorithm for different input rankings, then the scores given by the unsupervised metrics should be negatively correlated with the scores given by MAP. We can say that the more negative the correlation, the better the unsupervised distance metric.

We use this correlation analysis to compare the performances of the unsupervised evaluation measures. Results indicate that our modification makes Kendall Tau Distance more correlated with MAP. The results are shown in Table 1, KTDispSq is more negatively correlated for almost all rank aggregation methods we experimented. For two experiments KTDispSq is second to Canberra, but the differences are small in those cases. For the remaining experiments, KTDispSq outperforms others by large margins. This suggests that our variant makes Kendall Tau more closer to the supervised evaluation metric Mean Average Precision.

Table 1. Correlations with MAP for LETOR MQ2007 dataset. More negative is better.

\begin{tabular}{|l||c|c|c|c|}
\hline & Borda & SumScore & MC4 & QSort \\
\hline KT & -0.015 & -0.112 & -0.129 & -0.126 \\
\hline KTDispSq & $-\mathbf{0 . 0 5 8}$ & -0.123 & -0.136 & $\mathbf{- 0 . 1 3 4}$ \\
\hline SF & -0.020 & -0.109 & -0.124 & -0.118 \\
\hline Canberra & -0.041 & $\mathbf{- 0 . 1 2 4}$ & $\mathbf{- 0 . 1 3 7}$ & -0.127 \\
\hline PosWtD & 0.031 & 0.240 & 0.041 & 0.049 \\
\hline
\end{tabular}




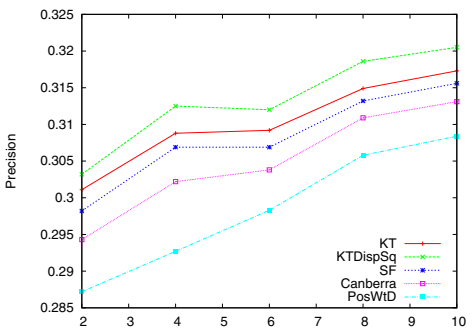

(a) Results for Precision

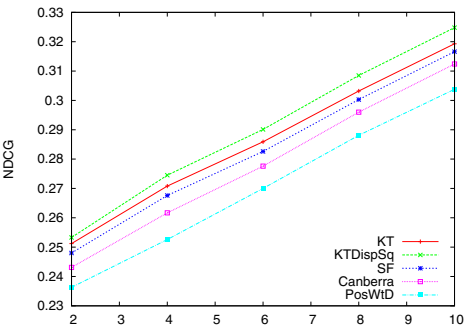

(b) Results for NDCG

Fig. 1. Improvement in Precision and NDCG by picking the best expert according to minimum average value of the measures. Higher values are better.

\subsection{Pick the Best Expert}

In the second set of experiments, we tried to see whether our modifications enable us to get better output for a rank aggregation task. For this, we took as experts some widely used features from the test datasets. For each query, we pick the expert which is closest to the remaining experts according to the metric value. Mathematically, if $R_{1}, R_{2}, \cdots, R_{n}$ are the input rankings, then we output as aggregate result $R_{i}$ such that $i=\arg \min _{i} \frac{1}{n-1} \sum_{k=1}^{n} \operatorname{dist}\left(R_{i}, R_{k}\right)$. Here dist represents the distance metric used for selecting the best expert. Features 1, 11, $15,16,20,21,40,41,45$ were used as experts for these experiments. The features correspond to scores of documents based on $t f$, $t f-i d f$, BM25, Language Model scores. Such features are often used for evaluating rank aggregation results 7 . Details of the features can be seen from [6].

Figure 1 displays graphically the Precision and NDCG values for the experiments. We showed Precision values for rank positions 2,4,6,8 and 10. As the

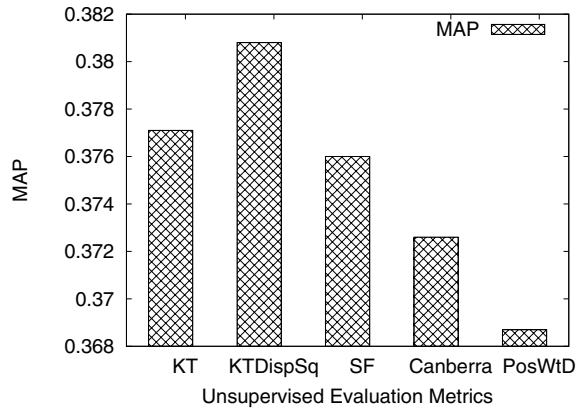

(a) Results for MAP

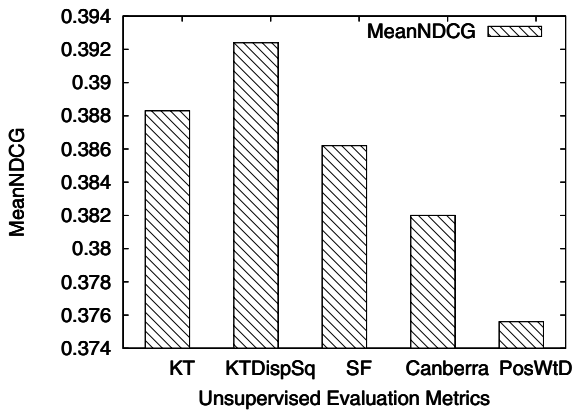

(b) Results for MeanNDCG

Fig. 2. Improvement in MAP and MeanNDCG by picking the best expert according to minimum average value of the measures. Higher values are better. 
graph suggests, the average Precision and NDCG scores obtained by selecting the lists using our measure are higher than that obtained by using other methods.

We also compared the values of MAP and MeanNDCG for this experiment. Results are shown in Figure 2. For both the measures, higher values indicate better performance. Here also we see that the ranked lists selected by using our measure obtain better scores than others. The results seem to indicate the efficacy of the proposed measure.

\section{Conclusions and Future Work}

We proposed a displacement based variant of Kendall Tau Distance for evaluating aggregated ranking. We can say that displacement based modification in Kendall Tau Distance makes it closer to other supervised metrics such as Precision and NDCG. Using KTDispSq in rank aggregation approach gives better Precision and NDCG, we can conclude that use of KTDispSq in place of Kendall Tau Distance may improve Rank Aggregation results. We plan to take into consideration position of displacement/inversion of document pair. Displacement at upper ranks should get penalized more than that of lower ranks. We would like to devise an unsupervised rank aggregation algorithm which directly optimizes this metric to generate the aggregated ranking.

\section{References}

1. Belkin, N.J., Kantor, P., Fox, E.A., Shaw, J.A.: Combining the evidence of multiple query representations for information retrieval. In: TREC 1995, Elmsford, NY, USA, pp. 431-448. Pergamon Press, Inc., Oxford (1995)

2. Carterette, B.: On rank correlation and the distance between rankings. In: SIGIR 2009, pp. 436-443. ACM Press, New York (2009)

3. Dwork, C., Kumar, R., Naor, M., Sivakumar, D.: Rank aggregation methods for the web. In: WWW 2001, pp. 613-622 (2001)

4. Jurman, G., Riccadonna, S., Visintainer, R., Furlanello, C.: Canberra distance on ranked lists. In: Proceedings, Advances in Ranking NIPS 2009 Workshop, pp. 22-27 (2009)

5. Kumar, R., Vassilvitskii, S.: Generalized distances between rankings. In: WWW 2010, pp. 571-580 (2010)

6. T.-y. Liu, J., Xu, T., Qin, W., Xiong, H.: Li: Letor: Benchmark dataset for research on learning to rank for information retrieval. In: SIGIR 2007 Workshop on Learning to Rank for Information Retrieval (2007)

7. Liu, Y., Liu, T.-Y., Qin, T., Ma, Z., Li, H.: Supervised rank aggregation. In: WWW, pp. 481-490 (2007)

8. Sanderson, M., Soboroff, I.: Problems with kendall's tau. In: SIGIR 2007, pp. 839$840(2007)$

9. Schalekamp, F., van Zuylen, A.: Rank aggregation: Together we're strong. In: ALENEX, pp. 38-51 (2009)

10. Yilmaz, E., Aslam, J.A., Robertson, S.: A new rank correlation coefficient for information retrieval. In: SIGIR 2008, pp. 587-594 (2008) 